\section{Christof Schnürer}

\section{Asthma und COPD: \\ Paradigma im Wandel}

Die obstruktiven Atemwegserkrankungen (OAW) werden seit ca. 3 Jahrzehnten zwei voneinander abzugrenzenden Krankheitsentitäten zugeordnet. Dies findet seinen Ausdruck in getrennten Leitlinien nationaler Fachgesellschaften und in den Empfehlungen zweier internationaler Initiativkomitees (GINA für Asthma [1] und GOLD für chronisch obstruktive Lungenerkrankungen (COPD) [2]). In der Alltagsrealität allerdings zeigte sich, dass weder die vorgegebenen Therapiealgorithmen noch die Diagnosezuordnungen konsequent umgesetzt werden (konnten). In den letzten Jahren wurden auch deshalb die Empfehlungen teils erheblich revidiert (GOLD zuletzt für 2017) und 2015 erstmals von den Initiativen gemeinsam eine «überlappende», beide Krankheitsdefinitionen erfüllende Fallgruppe (ACOS) eingeführt $[1,2]$. Bezüglich der Frage, was sich hinter «ACOS» verbirgt (Häufigkeit, Untergruppen, optimale Therapie?), besteht ein erhebliches Forschungsdefizit. Wenn sich herausstellen sollte, dass in den Studien, auf deren Aussagen die Konsensus-Dokumente beruhen, statistisch relevante ACOSGruppen nicht korrekt zugeordnet wurden, müssten die Leitlinien umgeschrieben werden.

\title{
Asthma - COPD - ACOS: Gesichtspunkte aus der Perspektive der Anthroposophischen Medizin
}

\section{Vereinheitlichende und dynamische Betrachtung der obstruktiven \\ Atemwegserkrankungen}

Das Grundproblem besteht darin, dass die den Studien zugrunde liegenden Krankheitsmodelle von weitgehend statischen Vorstellungen ausgehen. Die Wirklichkeit der OAW aber ist dynamisch, oft von Übergängen und im Verlauf wechselnden Zuständen geprägt. Darüber hinaus werden unter COPD zwei unterschiedliche Krankheiten («Phänotypen») subsumiert, die nur selten differenziert behandelt werden (Abb. 1). Vom Autor dieses Beitrags wird deswegen seit vielen Jahren ein dynamisches Krankheitsmodell vorgeschlagen $[3,4]$, das sich an der «funktionellen Dreigliederung des menschlichen Organismus» [5, S. 233-526] orientiert. Im Rahmen eines so ausgerichteten, komplementären Ansatzes konnten bei den meisten Patienten rasche Verbesserungen sowie ein deutlicher und dauerhafter Rückgang (Beobachtungszeiten vielfach $>10$ Jahre, Einzelfälle $>20$ Jahre) des Medikamentenbedarfs und der stationären Aufenthalte erreicht werden. In einer vom Behandler unabhängigen, prospektiven zweijährigen Beobachtungsstudie [6] wurde der Trend bestätigt.

OAW sind durch Störungen von Atem-Rhythmus, -Dynamik und -Fluss sowie der Clearance gekennzeichnet. Allergien, Infekte des Atemtraktes (einschliesslich Nase und Nebenhöhlen!) bis hin zu Pneumonien, Umbauvor- gänge, Leistungs- und Bewegungseinschränkungen, Hypoxämie, HerzKreislauf-Erkrankungen, Reflux, Ernährungs- und Stoffwechselstörungen, Depressionen, Angstzustände und seelisch-geistiger Rückzug finden sich in unterschiedlichen Verschränkungen und wechselnder Ausprägung. Somit handelt es sich um ein komplexes Geflecht von Ursachen und Folgen, das nur schrittweise erfasst und unter Einbeziehung des Patienten (Einsicht, $\mathrm{Zu}$ stimmung, Selbstmanagement) und salutogenetischer Therapien angemessen behandelt werden kann.

Schulmedizinische Medikamente sind (meist) geeignet, Linderungen bis hin zur Beschwerdefreiheit zu erreichen. So ist zweifellos dem konsequenten Einsatz inhalativer Corticosteroide (ICS) ein dramatischer Rückgang schwerer und letaler Asthmaanfälle zu verdanken. Kritisch zu beurteilen bleiben allerdings: Dosierung, Rhythmus, Substanzwahl und Therapiedauer. Indizierte und nicht durchgeführte oder (zu) hoch dosierte Corticosteroide und/oder Antiobstruktiva bergen unnötige Komplikationsrisiken und Adherence-Probleme. Eskalations- und Deeskalationsstrategien sollten deswegen Bestandteil jeder Therapie und Patientenschulung sein $[1,2]$.

\section{Durch Anthroposophische Medizin erweiterte Therapiestrategien}

Bereits 1921 wurde von Rudolf Steiner eine chronische Wunde als Asthma-

\section{KARGER}

(C) 2017 S. Karger GmbH, Freiburg

Fax +497614520714 
ursache beschrieben [7], die zu einer nachfolgenden Überempfindlichkeit der Bronchien führt. Eine Pathophysiologie, die mit den heutigen Vorstellungen zu den OAW (chronische Entzündungen) übereinstimmt. Im Rahmen der Weiterentwicklung der Anthroposophischen Medizin wurden Therapieansätze entwickelt, die auf vier Ebenen («Wesensglieder») des menschlichen Seins wirksam sind: 1. das PhysikalischChemische (Mess-Wägbare), 2. das Lebendige (unter anderem Atmung, Ernährung, Wachstum, Reproduktion), 3. das Seelische (Bewusstsein), 4. das Geistige (Neuschöpfung, Intentionalität «Ich») [5, S. 43-97]. Nur einige wenige können hier in der gebotenen Kürze skizziert werden.

Die «asthmatische Geste» (Obstruktion) erhält ihre Physiognomie durch einen «fixierten Schreck» (Inspiration/Anspannung). Chronische Entzündungen können durch «unverdautes» Seelisches (z.B. Schockerlebnisse) und/oder «unverdaubare» Noxen (z.B. inhalativ) verursacht werden. Beides dürfte als Kofaktor auch für das Tabakproblem gelten. Die unzureichende Fähigkeit, die Welt zu «verdauen», ein Übermass an Belastungen (stofflich und seelisch) führt zu «Unverträglichkeitsreaktionen», die - genauso wie nicht überwundene Atemwegsinfekte - in chronische Entzündungen und dann in OAW übergehen und - getriggert durch Kofaktoren als «Exazerbationen» immer wieder aufflammen können. Therapeutische Ansätze: Stärkung der «Verdauung» auf allen Ebenen; Kunst- und Bewegungstherapien, Wärmeanregung/ Fieber, Bitterstoffe und Gerbsäuren. Im «Ich», der geistigen Ebene des Menschen, sind die individualisieren-

\section{Literatur}

1 www.ginaasthma.org.

2 www.copdgold.org.

3 Schnürer C: Mehr Luft - Atemwegserkrankungen verstehen und überwinden. Stuttgart, Freies Geistesleben, 2006.
}

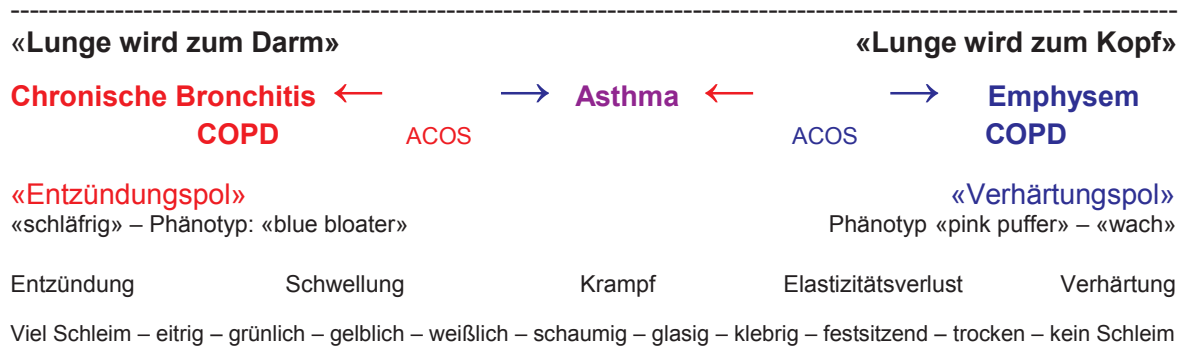

Abb. 1. Der Begriff COPD umfasst zwei polare Krankheitsbilder (Phänotypen). Asthma (bzw. die asthmatische Komponente einer COPD) hat beide polaren Tendenzen: chronische Entzündung und Verhärtung. Die Komponenten können in ihrem jeweiligen Anteil wechseln und können klinisch erfasst sowie differenziert behandelt werden. COPD ist nicht gleich COPD und Asthma nicht gleich Asthma. Für "ACOS» und für Asthma gelten: Der asthmatische Anteil (reversible Obstruktion) muss erfasst und gezielt («antiasthmatisch») behandelt werden. Dieser Anteil kann sich von Tag zu Tag und im Verlauf der Erkrankung - akut, vorübergehend, über längere Zeiträume - oder durch Medikamente in die schematisierten Pfeilrichtungen verschieben. Beispiel: Antibiotische Behandlung einer asthmatischen Exazerbation: Verschiebung vom Entzündungs- in den Verhärtungspol; der Schleim wird hell, zäh und schwer abhustbar (Krankheitsgeschehen wird im Schema nach rechts verlagert). Eine über Jahre bestehende oder/und schwere/unzureichend therapierte OAW führt zum progredienten «Zusammenrücken» beider Pole und zur Fixierung. Das Krankheitsbild «erstarrt» und wird immer weniger therapeutisch erreichbar (z.B. fixierte Obstruktion, Emphysem).

den, den «Krankheitstypus» überwindenden oder integrierenden Kräfte beheimatet. Seine Krankheit wird dem Patienten auf dieser Ebene «verstehbar» und zugänglich. Therapieansätze sind hier das Gespräch und Schulungen (Patientenexpertise, Selbstmanagement, eigenverantwortliche Deeskalation und Eskalation). Als Hilfsmittel haben sich Messungen des exspiratorischen Spitzenflusses (PEF) und Tagebücher bewährt. Alle aktivierenden Therapien beruhen letztlich auf der Ich-Ebene («ich will») und auf Rhythmus (Lebensebene): Heileurythmie, anthroposophische SprachSing-Atemtherapien, andere künstlerische Therapien, Sport, Meditation und nicht zuletzt der Nikotinentzug. Jede für sich allein, in Kombination und/oder in angemessener zeitlicher Abfolge kann hochgradig wirksam und für den Verlauf und Medikamentenbedarf entscheidend werden.

4 Schnürer C: Asthma und COPD. Menschenkundliche Grundlagen für eine rationale Diagnostik und Therapie. Merkurstab 2011;64:384391.

5 Selg P (Hrsg): Rudolf Steiner: Texte zur Medizin - Anthroposophie und Heilkunst. Teil I. Dornach, Rudolf Steiner, 2004.

\section{Funktionelle Dreigliederung und Krankheitstypus}

Das oben angesprochene Krankheitsmodell (schematische Darstellung in Abb. 1), abgeleitet aus der anthroposophisch erweiterten Physiologie, der «funktionellen Dreigliederung des menschlichen Organismus» [5, S. 233-526], hat sich bei dem Autor in der Überwindung des Schemas Asth$\mathrm{ma} / \mathrm{COPD}$ bewährt; es erfasst auch alle Überlappungen (ACOS) und ist so auch für den rationalen Einsatz schulmedizinischer Pharmaka geeignet.

Demnach lassen sich die Atemwegserkrankungen drei Grundtypen zuordnen, wobei (1) und (3) «polare» Krankheitsbilder sind:

(1) Chronische Bronchitis: Auflösung, Entzündung.

(2) Asthma: Entzündung und (meist anfallsweise) Verhärtung.

(3) Emphysem: Verhärtung, Erstarrung.
6 Hamre HJ, Witt CM, Kienle GS, et al: Anthroposophische Therapie für Asthma: Eine zweijährige prospektive Kohortenstudie in der routinemäßigen ambulanten Versorgung. Merkurstab 2011;64:426-442.

7 Steiner R: Vom Leben des Menschen und der Erde, ed 2. GA 349. Neunter Vortrag. Dornach, Rudolf Steiner, 1980, pp 165-167. 